\title{
Multipole moment decomposition for imaging hydraulic fractures from remote elastostatic data
}

\author{
B Lecampion $^{1,3}$ and A Peirce ${ }^{2}$ \\ ${ }^{1}$ CSIRO Petroleum, Melbourne, Australia \\ ${ }^{2}$ Institute of Applied Mathematics, University of British Columbia, Canada \\ E-mail: blecampion@clamart.oilfield.slb.com and peirce@math.ubc.ca
}

Received 12 March 2007, in final form 11 June 2007

Published 6 July 2007

Online at stacks.iop.org/IP/23/1641

\begin{abstract}
Hydraulic fracturing involves the propagation of a fracture in brittle rock by the intrusion of a high pressure viscous fluid. There is considerable interest in identifying characteristics of these evolving underground fractures via the passive monitoring of remote elastostatic deformations. In this paper, we present a far-field multipole expansion procedure to identify the harmonic moments of the fracture. The harmonic moments are related to fundamental quantities such as fracture volume and fracture asymmetries. We illustrate the efficacy of the multipole moment expansion technique by inverting synthetic displacement data from a hydraulic fracture simulator in order to identify the harmonic moments up to second order. These results are compared to those obtained by identifying the parameters of a dislocation model with a prescribed geometry - a procedure which is commonly used for such problems. The multipole moment expansion technique has the following features: it provides significantly more accurate fracture volume information; it provides accurate estimates of first-order moments that can be used to identify asymmetric fractures; it is possible to adapt the truncation process to optimize the information content of a given set of measurements; it can, in some cases, provide estimates of the higher order moments which can be used to determine geometric attributes of the fracture. Given this last feature, we explore the possibility of using up to second-order harmonic moments to identify the dimensions of a simple polygonal model of the fracture footprint. This procedure is tested by attempting to identify fracture footprints from synthesized hydraulic fracture data.
\end{abstract}

(Some figures in this article are in colour only in the electronic version)

3 Now with Schlumberger, 1 rue H Becquerel, 92142 Clamart, France. 


\section{Introduction}

Fluid-driven fractures are a class of tensile fractures that propagate in a brittle material as a result of the internal pressurization of the fracture by the injection of a viscous fluid. The material is typically subjected to pre-existing confining stresses-so that the fracture develops in the plane of least resistance normal to the minimum principal stress direction. Although such fractures occur naturally through magma-driven flows in the upper Earth's crust, hydraulic fracturing is frequently used to increase oil and gas reservoir permeability in order to enhance production [14].

In such hydraulic fracturing treatments, other than the measurement of the volume and pressure of the injected fluid, very little information about the extent of the evolving fracture surface is readily available. Such information is very important to enable engineers to avoid fracture breakout into environmentally sensitive regions or the loss of hydrocarbons. In order to improve the design of these fractures, considerable effort has been devoted to modelling the forward problem. This effort has ranged from analytic solutions [11] for simple geometries to sophisticated numerical models $[1,9,33,34]$ that solve the system of nonlinear integro-partial differential equations defined on domains with moving boundaries. The governing equations involve an integral equation expressing the force equilibrium between the fluid pressure and the elastic response of the rock represented by the crack width, the nonlinear lubrication equation that governs the fluid flow within the fracture cavity by enforcing the conservation of fluid volume, and a fracture propagation condition in which the fracture perimeter is located so that the stress intensity equals the local rock fracture toughness. Recent asymptotic analysis $[11,12,20,21,31]$ has demonstrated that these coupled equations have a complex multi-scale structure in which competing physical processes manifest at different length and time scales. Simplifying assumptions, such as a state of plane strain, required to derive these asymptotic solutions makes inversion using these asymptotic solutions too restrictive to yield useful information, while the formidable computational task of inversion using complex forward numerical models precludes real-time prediction of the fracture evolution.

In order to gain feedback about the evolving fracture there is, therefore, considerable interest in the passive monitoring of remote elastostatic deformations in order to identify characteristics of evolving fractures [16]. Such inverse problems are related to those that occur in non-destructive evaluation (NDE) $[5,6]$ in which quasi-static boundary loads are applied to an elastic body and the corresponding displacements are measured to detect cracks. For the passive monitoring problem the boundary loading on the crack is not known, while the small number of measurements that can feasibly be taken precludes the use of methods recently proposed for the NDE problem, such as the reciprocity gap functional $[2,3]$ or the method of topological derivatives [18].

The inverse problem is related to the elastic integral equation of the hydraulic fracturing problem, in which the fluid pressure is in force equilibrium with the crack width. The inverse problem aims to determine the crack width and geometry from remote displacement measurements elsewhere in the elastic medium. Consistent with St Venant's principle, the elasticity operator rapidly smoothes the details of the crack width and geometry with distance. Indeed, in [28] it is demonstrated that only the fracture volume and orientation can be identified if $\ell / r<2 / 3$, where $\ell$ is the characteristic length of the fracture and $r$ is the mean measurement distance to the centre of the fracture. As an illustration of the loss of geometric information, we observe that the deformation induced at remote points by a finite fracture is indistinguishable from that produced by an infinitesimal displacement discontinuity (DD) whose density equals the fracture volume. This severe non-uniqueness when using far-field observations has also been mentioned, although not fully explained, in the case of fracture detection from 
boundary measurements $[17,25,26]$. The solution of a similar linear inverse problem has also been considered by Hori [24] using a truncated spectral decomposition of the underlying Green's function with specially constructed eigenfunctions for measurement points that lie on a surface. The truncation in Hori's technique is based on the spectral content of the signal, while the technique we propose involves a truncation of unresolved moments in the far-field deformations. The spectral technique, which is computationally intensive, is not appropriate in the petroleum context in which real-time inversion is the ultimate objective and for which the combination of surface and interior measurements need to be synthesized.

The harmonic moment decomposition provides a class of far-field approximations in which arbitrary fracture geometries and boundary conditions can be represented. Moreover, the increasing hierarchy of moments corresponds to a sequence of multipoles of increasing order, in which deformation field due to the higher order multipoles decay more rapidly with distance. Thus the multipole expansion provides a natural separation of the contributions of each of the harmonic moments according to their radius of influence. Therefore, for a given set of measurements with a known signal-to-noise ratio, it is possible to determine the order of moments that can feasibly be identified. The advantage of this separation of moments is illustrated in the numerical experiments in which significantly more accurate fracture volume estimates are obtained compared to other inversion techniques that lump all the moment contributions. Multipole expansions [29, 35, 43] have also been used to great effect to yield fast ways to evaluate the crack boundary integral operator. Although the fast multipole method can be used to substantially accelerate the computations of our moment expansion method, we restrict our discussion to the accuracy of the moment expansion technique and not its most efficient implementation.

In section 2, we describe the inverse problem, introduce the moment expansion and use a simple example to illustrate the effect of truncating the expansion at different $\ell / r$ ratios. In section 3 , we use deformation time-series data obtained from a hydraulic fracture simulator for complex fracture geometries to explore the efficacy of the moment expansion method. Finally in section 4, we investigate the possibility of reconstructing the fracture shape from the knowledge of some of its harmonic moments. A simplified polygonal model for the fracture geometry with a constant opening is tested in order to simplify the problem. The examples considered are taken from situations typically encountered in the mapping of hydraulic fractures in the oil and gas industry.

\section{The forward and inverse problems}

\subsection{The inverse problem}

The hydraulic fractures we consider propagate primarily as mode I fractures corresponding to normal opening, while the elastic deformation measurements are sufficiently remote from the fracture for a homogenous elastic medium with an effective elastic modulus to be defined. We therefore consider deformations due to the opening of a planar fracture in a semi-infinite, homogeneous, isotropic, linear elastic medium. Since the location of the source of the fracture is known to be at the well-bore perforation, we assume that we know the location of the centre of the fracture. Hydraulic fractures typically take the line of least resistance and propagate in a plane that is perpendicular to the direction of the minimum principal in situ compressive stress. We therefore assume that the orientation of the fracture plane is known. We assume that displacements induced by the hydraulic fracture are monitored at points off the fracture surface [16]. Typical deformation data from tiltmeter measurements are located in monitoring wells adjacent to the fracture (see figure 1) or on the Earth's surface. In fact, 


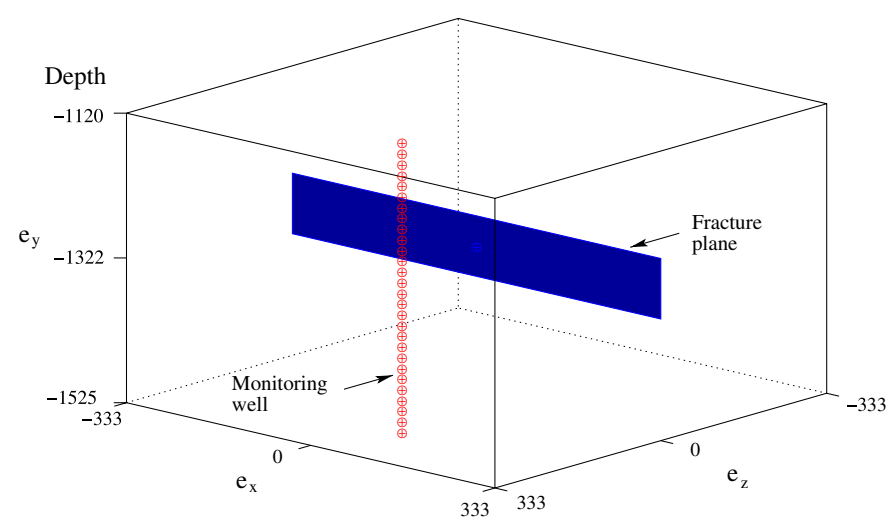

Figure 1. Typical location of downhole measurements in a monitoring well (dots) with respect to the fracture plane.

tiltmeters measure the horizontal components of the curl of the relative displacement vector that is induced by the presence of the fracture.

For ease of presentation we assume that the measurements comprise the displacements $u_{i}(\mathbf{x})$ induced by a planar fracture $S$ having a width distribution $w$. Such displacements are relative to the initial uncracked configuration. The governing integral equation is $[23,32]$

$$
u_{i}(\mathbf{x})=\int_{S} K_{i}\left(\mathbf{x}, \mathbf{x}^{\prime}\right) w\left(\mathbf{x}^{\prime}\right) \mathrm{d} \mathbf{x}^{\prime}
$$

where $K_{i}\left(\mathbf{x}, \mathbf{x}^{\prime}\right)$ denotes the fundamental displacement field at $\mathbf{x}$ due to a unit displacement discontinuity located at $\mathbf{x}^{\prime}$ within the planar region $S$. As stated above we have assumed that we know the orientation of the minimum principle stress so that the orientation of the fracture plane is known and that the measurement points $\mathbf{x}$ are located outside the fracture domain $S$ so that the integrand in (1) is regular.

The inverse problem is described by the Fredholm integral equation of the first kind (1) defined on an unknown planar region $S$ - a typically ill-posed problem. As mentioned in the introduction, the ellipticity of the $3 \mathrm{D}$ elasticity operator results in a rapid smoothing of fine-scale features of the fracture with distance. Indeed, if $\ell / r<2 / 3$ only the fracture volume and orientation can be reliably inverted, while for $\ell / r \ll 1$ the displacement field due to an infinitesimal displacement discontinuity having a density equal to the fracture volume, is indistinguishable from that of the fracture itself. The loss of uniqueness for remote measurements is thus apparent. Our main objective is to devise a robust procedure to determine fundamental characteristics of the fracture geometry $S$ and to investigate the effect of the location of the measurements relative to the fracture on the resolution of these characteristics.

Several approaches have been used to solve such an inverse problem. The fracture plane is often discretized to reduce equation (1) to a system of linear equations of the form $\mathbf{u}=\mathbf{A} \cdot \mathbf{w}$ subject to bound constraints $(\mathbf{w} \geqslant 0)[7,8,13,25,39,41]$. When the measurements are sampled far from the crack, severe non-uniqueness is characterized by a singular matrixsometimes having only one non-vanishing eigenvalue. This approach combined with a proper regularization scheme provides robust results only when the measurements are close to the fracture. In an alternative approach, the crack shape is parametrized and the inverse problem is solved as a nonlinear optimization problem: either using the shape sensitivity of the crack 
[4] or assuming a simple a priori fracture loading and geometry (e.g., circular, rectangular, elliptical, etc) and the parameters of the chosen model are identified [40]. The inversion of the displacements using a priori chosen fracture models has the advantage of simplicity. However, the chosen model may enforce an inappropriate boundary condition or the incorrect geometry, while still partially fitting the measured data, which can lead to aphysical results (see [27] for a discussion).

\subsection{Moment decomposition}

In practice, the location of the measurements is typically remote from the fracture which results in a loss of information. We therefore consider the possibility of solving an approximate version of the inverse problem in which we exploit the far-field asymptotic expansion of Green's function in the integral equation (1) in order to yield a series expansion in the harmonic moments of the problem. This far-field asymptotic expansion is appropriate for remote measurements and allows considerable flexibility in that it is possible to truncate the series in order to extract most of the available information from the data measured at a given location.

In order to derive the far-field moment expansion, let $\mathbf{x}_{0}$ be a reference point located in the fracture plane (i.e., $\left.z_{0}=0\right)$ and rewrite the kernel $K_{i}\left(\mathbf{x}^{\prime}-\mathbf{x}\right)$ in (1) as $K_{i}\left(\mathbf{x}^{\prime}-\mathbf{x}_{0}+\right.$ $\mathbf{x}_{0}-\mathbf{x}$ ). Using a Taylor expansion of the kernel function (assuming $\left\|\mathbf{x}_{0}-\mathbf{x}\right\| \gg\left\|\mathbf{x}_{0}-\mathbf{x}^{\prime}\right\|$ or equivalently $r \gg \ell$ ) we obtain

$u_{i}(\mathbf{x})=\sum_{k=0}^{\infty} \sum_{m=0}^{k} \frac{1}{(k-m) ! m !} \frac{\partial^{k} K_{i}\left(\mathbf{x}_{0}-\mathbf{x}\right)}{\partial x^{k-m} \partial y^{m}} \int_{S}\left(x^{\prime}-x_{0}\right)^{k-m}\left(y^{\prime}-y_{0}\right)^{m} w\left(x^{\prime}, y^{\prime}\right) \mathrm{d} x^{\prime} \mathrm{d} y^{\prime}$.

For simplicity, these displacements are expressed in an orthonormal system of coordinates $\left(\mathbf{e}_{x}, \mathbf{e}_{y}, \mathbf{e}_{z}\right)$ with the basis vectors $\left(\mathbf{e}_{x}, \mathbf{e}_{y}\right)$ located in the fracture plane (see figure 1). The expansion (2) can be truncated at order $k$ to furnish a simple forward model. The unknowns are then the weights of the far-field series (2) also known as the harmonic moments of the fracture denoted by $M_{x^{k-m}} y^{m}$ and defined as

$$
M_{x^{k-m} y^{m}}=\int_{S}\left(x^{\prime}-x_{0}\right)^{k-m}\left(y^{\prime}-y_{0}\right)^{m} w\left(x^{\prime}, y^{\prime}\right) \mathrm{d} x^{\prime} \mathrm{d} y^{\prime}
$$

while the kernel derivatives $\partial^{k} K_{i}\left(\mathbf{x}_{0}-\mathbf{x}\right) / \partial x^{k-m} \partial y^{m}$ represent the $i$ th displacement components due to the $k$ th-order multipoles. The zeroth-order moment $M_{0}=\int_{S} w\left(\mathbf{x}^{\prime}\right) \mathrm{d} \mathbf{x}^{\prime}$ corresponds to the fracture volume. The higher order moments are related to more and more complex characteristics of the fracture shape (asymmetry, larger extension along one axis etc). It is important to note that all the odd-order moments $(m, k-m=1,3,5, \ldots)$ vanish for a symmetric fracture (in both $S$ and $w$ ) if the reference point $\mathbf{x}_{0}$ corresponds to the fracture centre $\mathbf{x}_{\mathbf{c}}$.

The expansion (2) requires only the knowledge of the derivatives of the fundamental kernel $K_{i}$. In the case of full and half-space isotropic elastic media, these expressions can be obtained in analytical form. Inverting displacements using such a truncated expansion has several advantages: (i) the unknowns of the inverse problem are the harmonic moments of the fracture which provide fundamental characteristics of its shape and loading; (ii) it is flexible in the sense that more terms can be added if the measurements become closer to the fracture, enabling the resolution of more harmonic moments of the fracture; (iii) there is no a priori postulate on either the shape of the fracture or its loading. We only assume that the measurements are performed at points that are remote from the fracture. 


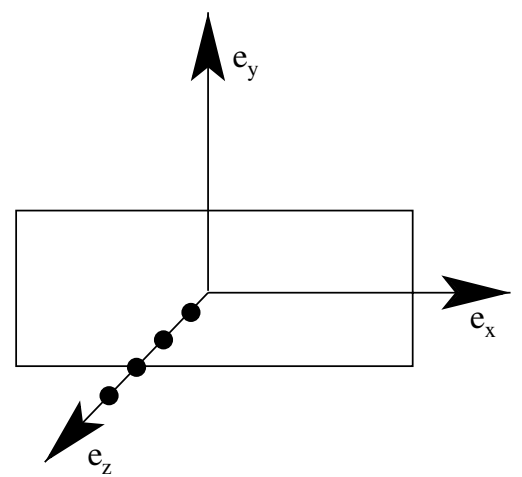

Figure 2. Forward example configuration, a rectangular displacement discontinuity with constant opening in an infinite medium.

Far and near field. We now derive a bound for the relative error in terms of the dimensionless ratio $\ell / r$. Firstly consider the remainder term in the Taylor expansion given as follows

$E_{k}=\frac{1}{(k+1) !} \sum_{m=0}^{k+1}\left(\frac{\prod_{j=k+2-m}^{k+1} j}{m !}\right) \frac{\partial^{k+1} K}{\partial x^{k+1-m} \partial y^{m}} \int_{S}\left(x^{\prime}-x_{0}\right)^{k+1-m}\left(y^{\prime}-y_{0}\right)^{m} w\left(x^{\prime}, y^{\prime}\right) \mathrm{d} x^{\prime} \mathrm{d} y^{\prime}$

We introduce the fracture length scale $\ell$ which is such that $\left|x^{\prime}-x_{0}\right|<\ell$ and $\left|y^{\prime}-y_{0}\right|<\ell$ for all $\left(x^{\prime}, y^{\prime}\right) \in S$. In addition, since the displacement kernel decays like $\frac{1}{r^{2}}$ [30], we introduce the following notation $\sum_{m=0}^{k+1}\left(\frac{\prod_{j=k+2-m}^{k+1} j}{m !}\right) \frac{\partial^{k+1} K}{\partial x^{k+1-m} \partial y^{m}}<\frac{C_{k}}{r^{k+1+2}}$ to obtain the following estimate for $E_{k}$

$$
E_{k}<\frac{C_{k+1}}{(k+1) !}\left(\frac{\ell}{r}\right)^{k+1} \frac{M_{0}}{r^{2}}
$$

Now if we specify a relative error tolerance of $\varepsilon_{k}$ with respect to the zeroth order

$$
\frac{E_{k}}{\frac{M_{0}}{r^{2}}}<\frac{C_{k}}{(k+1) !}\left(\frac{\ell}{r}\right)^{k+1}<\varepsilon_{k}
$$

we obtain the following restriction on the dimensionless ratio $\ell / r$ for a given truncation order $k$ :

$$
\frac{\ell}{r}<\left(\frac{\varepsilon_{k}(k+1) !}{C_{k}}\right)^{\frac{1}{k+1}}=\alpha_{k}
$$

This monotonically increasing sequence $\left\{\alpha_{k}\right\}$, for which $\alpha_{k} \rightarrow \infty$ as $k \rightarrow \infty$, defines the domain of validity of each successive order of the moment expansion. For example, it has been established [28] that for a prescribed maximum relative error of approximately 4-5\% the validity bound for the zeroth-order approximation is $\alpha_{0} \approx 0.8$ [28]. When the observation point is located closer to the fracture, the effect of the shape and loading becomes a significant component in the displacement field, and more terms in the series (2) are needed to reproduce the recorded displacement correctly.

We illustrate the effect of truncating the expansion (2) at different orders by a simple example. As our model crack, we assume a constant rectangular displacement discontinuity (or equivalently a rectangular crack with a constant opening) in an infinite elastic medium [36]. The configuration is sketched in figure 2. For different ratios $\ell / r$, we compare the $z$ displacement component induced by this displacement discontinuity (DD) to those of the 

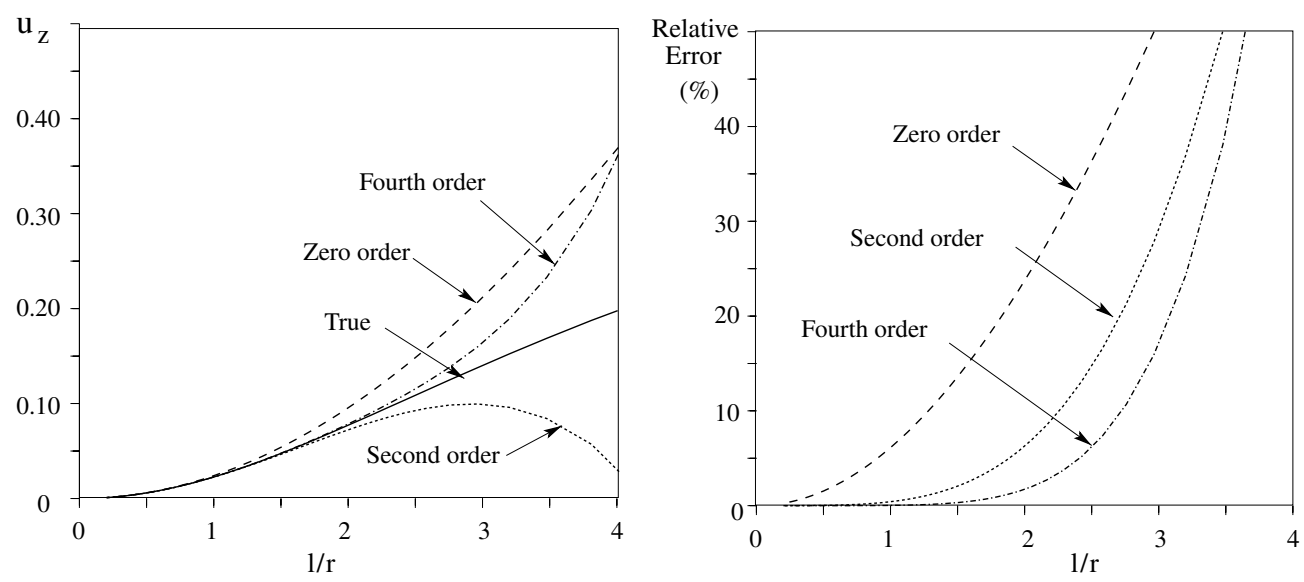

Figure 3. Evolution of the $z$ component of the displacement with the dimensionless distance $\ell / r$ (left); and relative error of the moment approximations compared to the correct solution comprising a unit rectangular displacement discontinuity (right).

zeroth-, second- and fourth-order moment expansions. In figure 3 (left), we compare the exact DD displacements to the displacements calculated using the zeroth-order, the secondorder $(k=4)$ and the fourth-order moment expansions $(k=8)$. In figure 3 (right), we plot the corresponding relative errors. It can be seen that the higher order expansions have stronger singularities that dominate closer to the fracture $\ell / r \gg 1$. Below the far-field limit $\ell / r<0.8=\alpha_{0}$, the zeroth-order expansion reproduces the displacement with an error of less than $4 \%$. For the same tolerance of $4 \%$, the second-order expansion is still valid for $\ell / r \approx 1.75=\alpha_{2}$ while the fourth-order expansion is valid for $\ell / r \approx 2.2=\alpha_{4}$.

\section{Synthetic examples}

In this section, we investigate the inversion of displacements induced by a pressurized fracture of known orientation using the moment decomposition presented in the previous section. The displacement data for a sequence of complex fracture geometries are generated using the numerical hydraulic fracturing (HF) simulator described in [34, 37]. Since the synthetic displacements are obtained numerically, some inherent noise is introduced due to truncation and round-off errors; however, no additional noise is added. The effect of noisy data has been studied [27] for the same class of problem but restricted to a first-order moment decomposition. Since the effect of noise on the method presented here does not produce significantly different results from those presented in [27], we do not consider the influence of noise in the discussion that follows. The 'measurements' are sampled along a vertical array of sensors (see figure 1), and we assume that only the $y$ component of the displacement field is recorded. The displacement data are simulated at different stages of the growth of the hydraulic fracture, which therefore produces both far-field and near-field conditions as the ratio $\ell / r$ increases with the growth of the fracture.

Different orders of expansion (2) and assumptions are investigated as forward models: (i) a zeroth-order expansion; (ii) a second-order expansion with zero first-order moments $M_{x}$; (iii) a complete second-order expansion; (iv) and a fourth-order decomposition with zero first- and third-order moments. The injection point is always taken as the reference point $\mathbf{x}_{0}$. The different hypotheses on the value of the first-order moment $M_{x}$ (zero or not) directly 


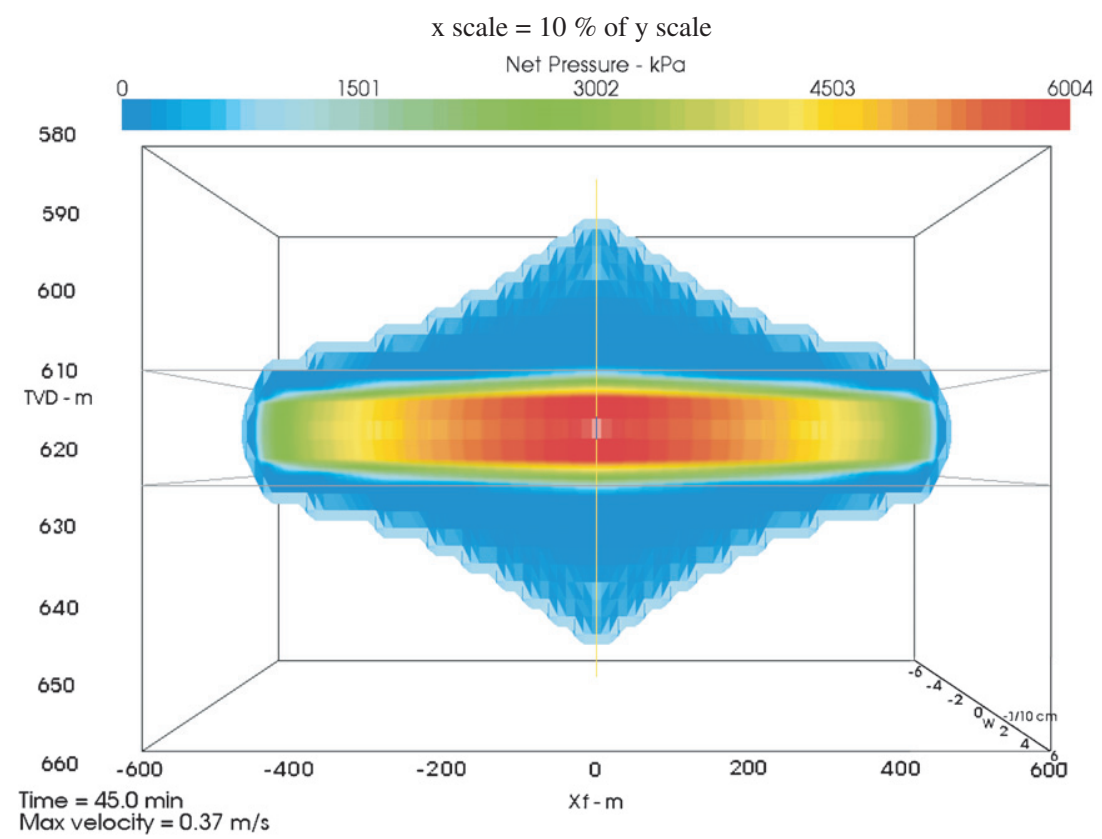

Figure 4. Symmetric height growth: fracture footprint (opening profile).

corresponds to different assumptions on the symmetry of the fracture. Since finite dislocations of a prescribed shape and density variation are frequently used in practice as inversion models $[40,42]$, we also consider the identification of the parameters for a finite rectangular constant displacement discontinuity (DD) for comparison.

The unknowns of the inverse problem are the harmonic moments $\mathbf{M}$ of the decomposition used. The solution of the inverse problem consists in minimizing the functional

$$
\begin{aligned}
& \mathcal{J}(\mathbf{M})=\frac{1}{2}\left\|u_{d}-u(\mathbf{M})\right\|_{2}^{2} \\
& \text { with } \quad M_{x^{n} y^{m}} \geqslant 0, \quad \text { for } n \text { and } m \text { odds }
\end{aligned}
$$

$\mathcal{J}(\mathbf{M})$ is the $\ell_{2}$ distance between the measurements $u_{d}$ and the predictions $u(\mathbf{M})$. The predictions are obtained by the truncation of the moment decomposition (2) at a given order. Due to the linearity of the decomposition (2) with respect to the moments, the problem is in fact a simple linear least-square fit with bound constraints. The positive constraints on the odd-order moments $\left(M_{0}, M_{x x}, M_{x x y y}\right.$ etc) are related to the requirement of a positive fracture aperture $w$ (i.e., no inter-penetration of the crack surfaces is permitted). The moment decomposition model uses analytic expressions for the different partial derivatives of the halfspace point force solution [30,32] obtained via Mathematica. Such a forward model is completely independent of the numerical HF simulator used to generate the examples data. We have also deliberately omitted any regularization terms in the minimization of (5) in order to focus the following discussion on the accuracy of the moment decomposition.

\subsection{A hydraulic fracture with symmetric height growth}

The first synthetic example investigates the case of a hydraulic fracture growing primarily within a horizontal layer bounded above and below by layers subjected to slightly higher confining stresses. As the fracture progresses it is not restricted to the middle layer with the 

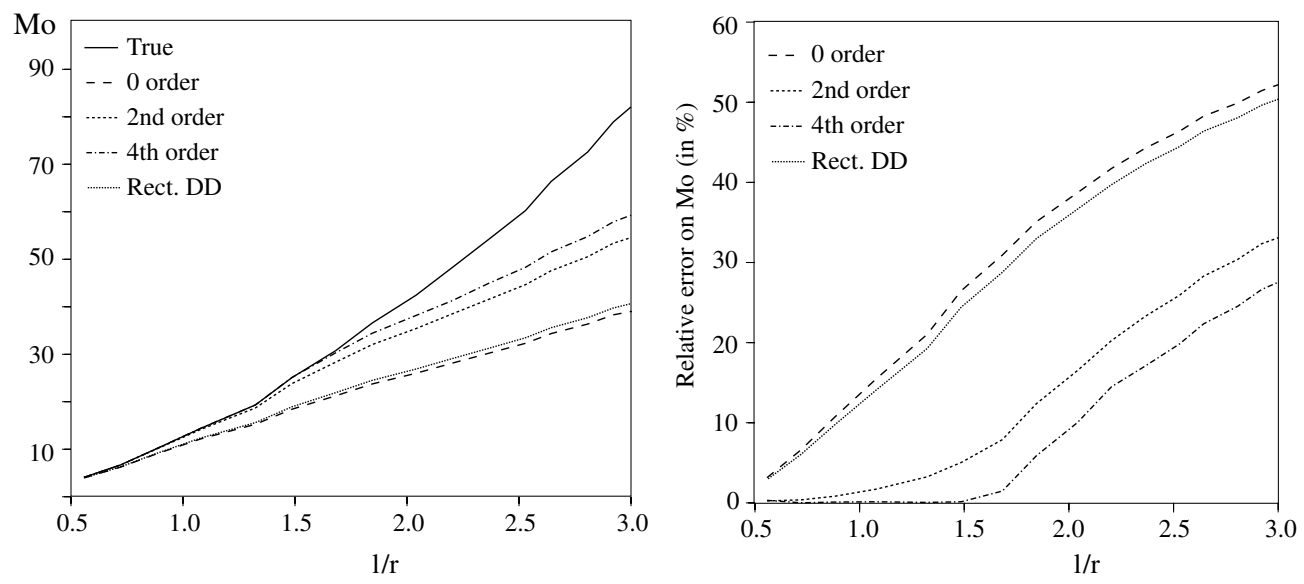

Figure 5. Symmetric height growth: fracture volume (zeroth-order moment $M_{0}$ ) of a symmetric PKN fracture with height growth estimated for different values of the distance ratio $\ell / r$ using a finite rectangular displacement discontinuity model, and moment decompositions of different orders (estimated volume on the left, relative error on the right).

lower confining stress, but, due to the moderate stress contrast, the fracture is able to penetrate the upper and lower neighbouring layers symmetrically (see figure 4). The height of the low confinement layer is $15 \mathrm{~m}$ while the maximum fracture height at the well-bore is approximately $65 \mathrm{~m}$. The fracture half-length (dimension along the $x$ axis) reaches a maximum of $550 \mathrm{~m}$.

In figure 5, we plot the estimated fracture volumes (i.e., the zeroth-order moment $M_{0}$ ) obtained using the different orders of moments. The results are all displayed for different values of the mean ratio $\ell / r$ which is calculated by averaging over the spatially distributed measurement points that remain fixed in space throughout the simulation. The variation in $\ell / r$ is due to the increase in the characteristic dimension of the fracture as the fracture evolves. In this case, we take the characteristic length $\ell$ to be equal to $\left(\ell_{x}+\ell_{y}\right) / 2$, where $\ell_{x}$ and $\ell_{y}$ are the horizontal and vertical fracture dimensions. The zeroth-, second- and fourth-order expansions correctly estimate (within 10\%) the fracture volume up to their corresponding limit of validity ( $\alpha_{0} \approx 0.8, \alpha_{2} \approx 1.75$ and $\alpha_{4} \approx 2$, respectively).

From figure 5, it can be seen that the fracture volume obtained using a finite rectangular $\mathrm{DD}$ is essentially equivalent to the result obtained using the zeroth-order moment expansion and is also valid for $\ell / r \lesssim 1$. We observe that this finite displacement discontinuity model is equivalent to an infinite series of moments in which the higher order terms are different from those of the actual hydraulic fracture in that the width is assumed to be constant as opposed to variable and the fracture footprint is assumed to be rectangular as opposed to arbitrarily shaped. The volume estimates for the constant displacement discontinuity model are inaccurate for higher $\ell / r$ ratios because there is a mismatch in the higher order terms of the constant displacement discontinuity moment expansion and the moment expansion for the actual fracture. As $\ell / r$ is increased, this discrepancy in the higher order terms starts to dominate the zeroth-order term. This clearly illustrates the limitation of such geometric models since they can only hope to capture the zeroth-order moment of the true solution.

An inversion model based on estimating the terms of the moment expansion (2) does not suffer from this limitation, since the addition of higher order terms can be used to remedy the problem of slow convergence associated with higher $\ell / r$ ratios. Indeed, the improvement of the estimate for $M_{0}$ seen in figure 5 , when the second- and then fourth-order moments are 

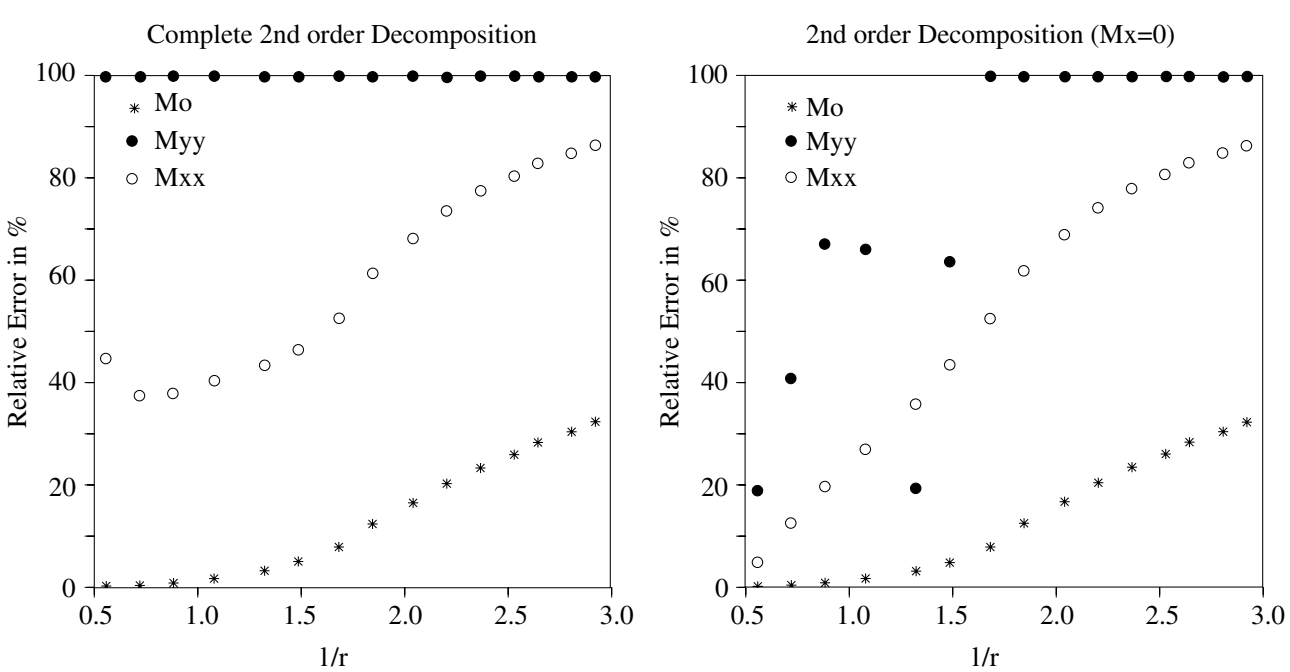

4th order Decomposition

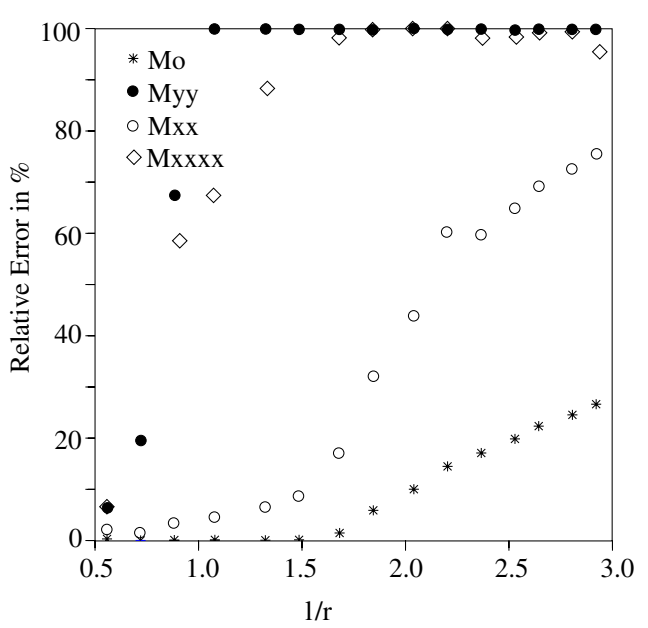

Figure 6. Symmetric height growth: evolution of the relative error on nonzero moments with the distance ratio $\ell / r$ for different orders of decomposition.

included, clearly demonstrates the benefit of correctly identifying the higher order moments so that their contribution is not spuriously added to the zeroth-order moment. We also note from figure 5 that the second-order approximations with different assumptions on the firstorder moments yield essentially the same estimate of the zeroth-order moment. Thus, in the estimation of the zeroth-order moment, the important issue is to separate the contributions of the zeroth-order moment from the higher order ones, rather than to identify the detailed split of the remaining contribution between the higher order terms.

In figure 6, the estimates of the nonzero second-order moments obtained from the different expansions are compared to the correct values computed from the synthetic data. More precisely, we plot the relative errors of the estimated moments, while table 1 contains actual numerical values for the second-order moments $M_{x x}$ and $M_{y y}$. The second-order moment estimates deteriorate rapidly as $\ell / r$ increases, particularly for values above $\ell / r \approx 1.5$ 
Table 1. Symmetric height growth: comparisons of the estimated second-order moments $M_{x x}$ and $M_{y y}$ obtained using the second- and fourth-order decompositions for different ratios $\ell_{y} / \ell_{x}$.

\begin{tabular}{|c|c|c|c|c|c|c|c|}
\hline \multirow[b]{2}{*}{$\ell / r$} & \multirow[b]{2}{*}{$\ell_{y} / \ell_{x}$} & \multicolumn{3}{|c|}{$M_{x x}$} & \multicolumn{3}{|c|}{$M_{y y}$} \\
\hline & & True & Second $\left(M_{x}=0\right)$ & Fourth & True & Second $\left(M_{x}=0\right)$ & Fourth \\
\hline 0.557 & 0.182 & $3.65 \times 10^{3}$ & $3.47 \times 10^{3}$ & $3.72 \times 10^{3}$ & $6.04 \times 10^{1}$ & $7.182 \times 10^{1}$ & $6.42 \times 10^{1}$ \\
\hline 0.720 & 0.130 & $1.22 \times 10^{4}$ & $1.06 \times 10^{4}$ & $1.20 \times 10^{4}$ & $1.04 \times 10^{2}$ & $1.467 \times 10^{2}$ & $8.38 \times 10^{1}$ \\
\hline 0.882 & 0.101 & $3.23 \times 10^{4}$ & $2.59 \times 10^{4}$ & $3.12 \times 10^{4}$ & $1.66 \times 10^{2}$ & $2.786 \times 10^{2}$ & $5.44 \times 10^{1}$ \\
\hline 1.07 & 0.101 & $7.05 \times 10^{4}$ & $5.15 \times 10^{4}$ & $6.73 \times 10^{4}$ & $2.597 \times 10^{2}$ & $4.310 \times 10^{2}$ & $7.302 \times 10^{-3}$ \\
\hline 1.32 & 0.0799 & $1.41 \times 10^{5}$ & $9.06 \times 10^{4}$ & $1.31 \times 10^{5}$ & $3.73 \times 10^{2}$ & $4.46 \times 10^{2}$ & $6.51 \times 10^{-3}$ \\
\hline 1.48 & 0.0700 & $2.54 \times 10^{5}$ & $1.43 \times 10^{5}$ & $2.32 \times 10^{5}$ & $5.14 \times 10^{2}$ & $1.87 \times 10^{2}$ & $7.48 \times 10^{-3}$ \\
\hline 1.68 & 0.0735 & $3.95 \times 10^{5}$ & $1.88 \times 10^{5}$ & $3.27 \times 10^{5}$ & $6.84 \times 10^{2}$ & $5.23 \times 10^{-8}$ & $7.053 \times 10^{-3}$ \\
\hline 1.84 & 0.0661 & $5.73 \times 10^{5}$ & $2.19 \times 10^{5}$ & $3.89 \times 10^{5}$ & $8.77 \times 10^{2}$ & $3.91 \times 10^{-8}$ & $6.844 \times 10^{-3}$ \\
\hline
\end{tabular}

(see figure 6). When the complete second-order approximation is used (without any a priori assumption on the first-order moments), it does not recover the second-order moments accurately even for low values $\ell / r$. The first-order moments are not zero (whereas they should be for this symmetric case) which affects the accuracy of the estimated second-order moments. When the first moment $M_{x}$ is forced to be zero the estimate improves - at least for low values of $\ell / r$. The results are even better when the fourth-order approximation is used, again only for $\ell / r \lesssim 1.5$.

It is also clear that, independent of the order of the decomposition, $M_{x x}$ is estimated more accurately than $M_{y y}$ (at least for values of $\ell / r \lesssim 1$ ), which is related to a better resolution of the fracture length than the fracture height. This is consistent with a fracture extending largely in the $x$ direction (for a maximum of $1100 \mathrm{~m}$ for the total fracture length) but remaining narrow in the $y$ direction (see figure 4). From table 1 , we see that as soon as the ratio $\ell_{y} / \ell_{x}$ falls below 0.1 , the estimates of $M_{y y}$ become very poor. The resolution of second-order moments is also closely linked to the aspect ratio of the fracture $\ell_{y} / \ell_{x}$. For a fracture with such an extreme aspect ratio the $x$ second-order moment $M_{x x}$ is two orders of magnitude larger than $M_{y y}$. Although, the absolute error in the estimation of these disparate moments may be the same, the relative errors render the estimation of the smaller moment to be meaningless.

Repetitive inversion. It is interesting to note that the fit of the displacement data obtained with the different models for a near-field case $(\ell / r=2.46)$ all have the same minimum residual errors. For such large values of $\ell / r$, all volume estimates obtained are inaccurate (see figure 5), while the predicted displacements are nearly indistinguishable from the measured data. This clearly illustrates the non-uniqueness that is typical of such ill-conditioned inverse problems. Moreover, in practical situations, we do not know the fracture length-scale $\ell$ so that it is impossible to know if a particular approximation is valid or not. The use of several moment decompositions to invert the data can overcome this problem. Indeed, for a given data set, the analysis can be performed repetitively using the zeroth-, second-, etc, up to $k$ th-order expansions. The estimates of the harmonic moments obtained from these different approximations can then be compared in order to obtain an estimate of $\ell / r$. For example, if the zeroth-order moment obtained via the second- and fourth-order expansions are similar to, but different from, the estimate obtained using the zeroth-order approximation, it indicates that the zeroth-order approximation is no longer valid: i.e., $\ell / r \gtrsim 1$. This procedure can be repeated for the higher order moments $\left(M_{x}, M_{y}, M_{x x}, \ldots\right)$ using the higher order approximations in order to refine the bound on $\ell / r$. 


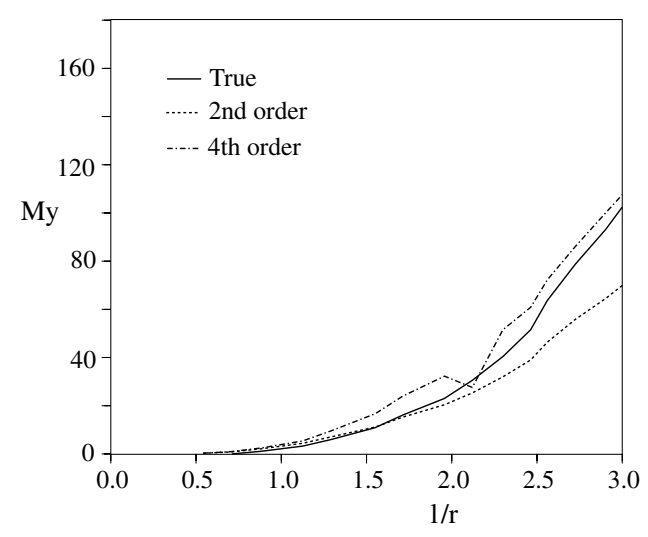

Figure 7. Asymmetric height growth: estimates of $M_{y}$ with respect to $\ell / r$.

\subsection{A hydraulic fracture with asymmetric height growth}

We now consider a more complex asymmetric fracture geometry. The hydraulic fracture is assumed to grow primarily within a horizontal layer bounded above by a layer with a slightly higher confining stress and below by a layer with a significantly higher confining stress. As the fracture progresses it breaks into the upper layer while it is restricted from breaking into the lower layer by the high confinement. Similar to the previous case, the fracture volume is estimated correctly using the different orders of moment decomposition up to their limit of validity. The conclusions regarding the zeroth-order and second-order moment estimates are similar to those for the symmetric case. It is interesting to compare the estimated first-order moment $M_{y}$ with the correct value (see figure 7). The development of the vertical asymmetry of the fracture is reflected in the deviation of the fracture centre from the injection point, so that the value of $M_{y}$ increases for higher $\ell / r$ or equivalently for larger fractures. The first-order moment $M_{y}$ is identified accurately even for higher values of $\ell / r$ (see figure 7 ), independent of the assumptions made on the other first-order moment $M_{x}$. The fourth-order approximation gives even better results. The accurate estimation of $M_{y}$ is a significant result as it indicates that asymmetry can be quantitatively detected from field measurements.

\section{Inversion of shape from moments}

It is possible to formulate a second class of inverse problem which involves determining the shape of the fracture from the values of a number of the harmonic moments. This classic inverse problem has received a lot of attention (see for example [22] and references therein). In our case, only a small number of moments can be determined, which makes it hard to apply some of the available algorithms. As a result we adopt an approach which makes it possible to use a lot of a priori information about the fracture geometry, which serves to constrain the problem and limit the number of degrees of freedom.

\subsection{Polygonal shape}

Hydraulic fractures typically found in oil and gas applications are mainly contained in one sedimentary layer (the target layer) but with possible 'break-out regions' into one or both of the neighbouring layers above and below the target layer (see figure 4). We therefore propose a simple polygonal shape as a geometric model for the fracture footprint and assume 


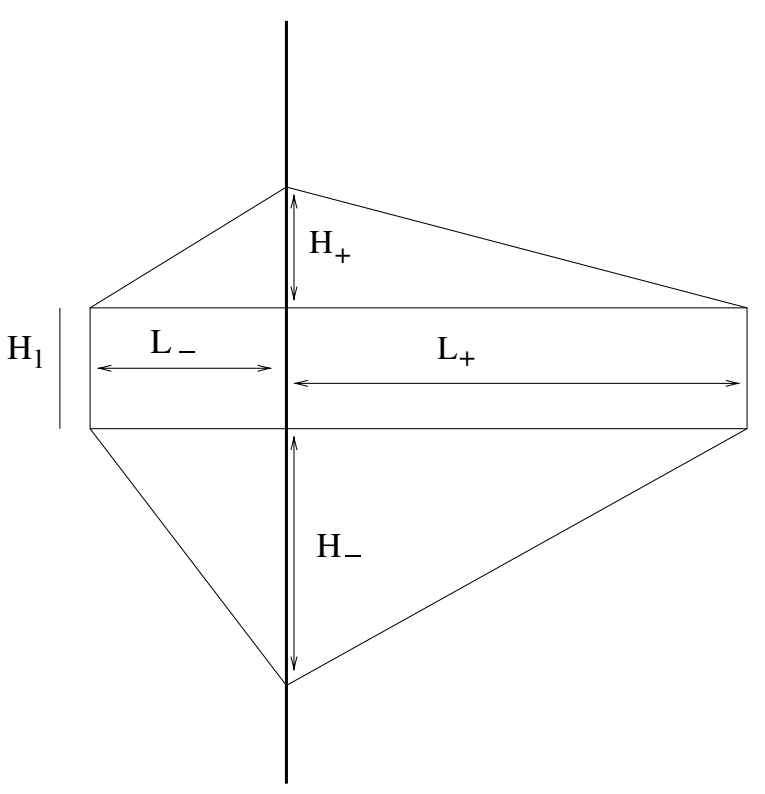

Figure 8. A polygonal geometric model of a fracture footprint with break-out typically encountered in the oil and gas industry.

a constant width for the fracture opening-although this latter approximation may appear somewhat extreme. The middle part of our proposed model of the fracture footprint comprises a rectangle of height $H_{l}$, which corresponds to the target layer. We allow for the possibility of asymmetric fracture growth in the horizontal and the vertical directions. The top and bottom parts of the fracture footprint model are approximated by triangles (see figure 8). Assuming a constant fracture opening $\bar{w}$, analytical expressions for the zeroth-, first-, and second-order moments of such polygonal models can be obtained in terms of the geometric parameters: $H_{l}, L_{+}, L_{-}, H_{+}, H_{-}$and $\bar{w}$. For example, $M_{0}^{p}=\bar{w} \int_{S} \mathrm{~d} x^{\prime} \mathrm{d} y^{\prime}=\frac{\bar{w}}{2}\left(L_{+}+L_{-}\right)\left(H_{+}+H_{-}+\right.$ $\left.2 H_{l}\right)$, etc. Naturally, other parametrizations of the geometry and the functional variation are possible, e.g., we might assume a fracture opening in the form of a bubble function with a square root tip behaviour. However, asymptotic analysis [11, 12, 20, 21, 31] has shown that the tip behaviour is multi-scale, in which a different power laws might be active along different parts of the periphery of the fracture. This will be difficult to parametrize with a few degrees of freedom. In addition, imposing an incorrect power law, which is more singular at the tip, might lead to more errors than the constant fracture opening assumption which will identify the average width level.

Using this approximate fracture shape and having some estimate of the harmonic moments, we are able to formulate a nonlinear regression problem in which the dimensions of the polygonal fracture model are the unknowns. We minimize the error between the harmonic moments of the fracture used to generate the synthetic data $\mathbf{M}$ and those of the polygonal model $\mathbf{M}^{p}$. More specifically, a Bayesian inversion procedure is used. Weak priors (i.e., flat prior probability density functions) are prescribed in order to reflect our ignorance of the fracture dimensions. An analysis of the curvature of the posterior probability density function makes it possible to estimate the posterior covariance matrix and therefore the correlation coefficients between the different parameters estimated (see [10] for details of this classical approximation in Bayesian inversion). Bound constraints are also prescribed on the mean 
opening: $0<\bar{w}<10 \mathrm{~mm}$. The minimization is performed using the so-called differential evolution algorithm, a particular genetic algorithm described in [38].

It is also possible to reformulate the inversion problem to obtain the assumed shape parameters directly from the displacement data, rather than our two-step approach in which the moments are determined first and then the shape parameters are identified from these moments. This alternative approach, should yield an equivalent sequence of moments, since the moments are intrinsic quantities which should not depend on the parametrization used. However, we have chosen to identify the moments directly since they are fundamental quantities which contain important practical information. For example, comparing the identified fracture volume, which is given directly by the zeroth-order moment, to the amount of fluid pumped into the fracture enables the field engineer to determine the so-called leak-off associated with the fracture. In addition, first moment provides useful information about the initiation of asymmetry — which could be used in field treatments to identify possible break-out.

\subsection{Results}

We investigate the hydraulic fracture with asymmetric height growth considered in the synthetic example of section 3.2. Firstly, in an attempt to test the method, we assume that we have accurate harmonic moments up to second order. Secondly, the effect of noisy data is also investigated by adding a Gaussian noise component to the accurate moments with a variance equal to $5 \%$ of the actual value. Finally, the same inversion procedure is repeated using the moments identified by inverting the displacement data as discussed in section 3. This procedure is repeated for different $\ell / r$ ratios for the asymmetric height growth situation. For the results that we present, the ratio $\ell / r$ is used to characterize the effect of truncating the moment expansion when determining the moments from the inversion of the synthetic displacement data. This ratio is not relevant for moments calculated directly (or those with 5\% noise) from the 'true' fracture footprint and width used to generate the synthetic displacement data since there are no measurement distances $r$ for these cases.

Figure 9 displays, for different $\ell / r$ ratios, the 'true' fracture footprint used to generate the synthetic data and the different estimated polygons. The 'true' fracture footprint is represented by coding the computational nodes having a positive opening with a black dot. The shape obtained with the perfect moments or the moments with synthetic noise are consistent with the actual footprint, although they do not fully capture the height growth as the fracture propagates. This can be partly explained by the fact that the top of the 'true' fracture footprint is not really a triangular shape: the part of the fracture on the top layer is more localized close to the well, while the chosen polygonal shape tends to spread the area of the upper part over the entire fracture length (see figure 4). The fracture symmetry with respect to the $y$ axis is, on the other hand, well captured by the polygon shape and the estimates of the fracture half-length are relatively good. As can be expected, the results obtained from the moments inverted from displacement data using the different orders of decomposition are less accurate, especially as the ratio $\ell / r$ increases. The asymmetric height growth is not well identified and the half-length is underestimated. It is not surprising that for a ratio $\ell / r$ above 1.5 the results are poor as this corresponds to the point where the harmonic moments are not well resolved by the inversion of displacement data.

Figure 10 compares the average aperture for the synthetic fracture with the different estimates of the constant aperture $\bar{w}$. The aperture estimated from the inverted moments has a tendency to lock on to the upper bound constraint early in the evolution of the fracture (see figure 10) whereas the corresponding fracture half-length is under-estimated (see figure 9). It is extremely important to point out that some values of the polygonal dimensions estimated 


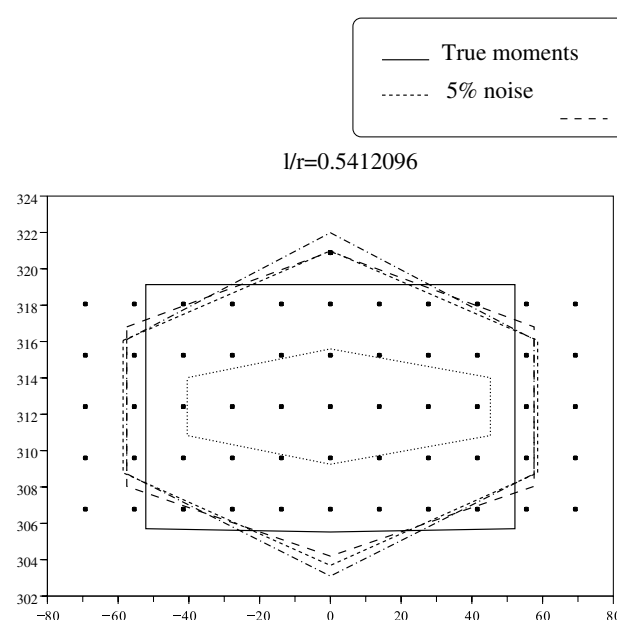

Estimated 2nd order

Estimated 2nd order $\mathrm{Mx}=0$ Estimated 4th order

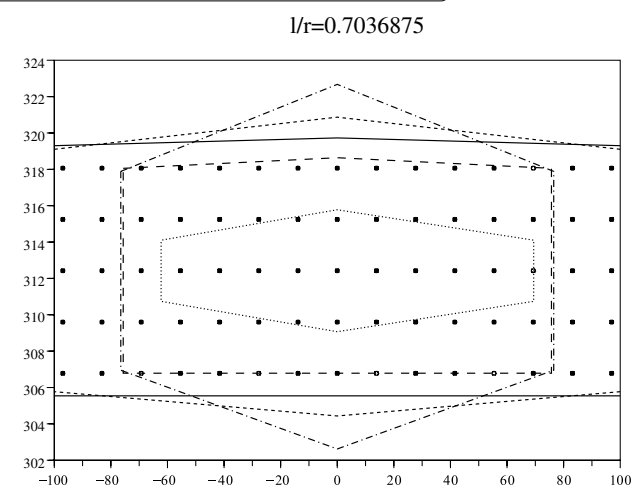

$1 / r=1.127029$

$1 / \mathrm{r}=1.5506109$
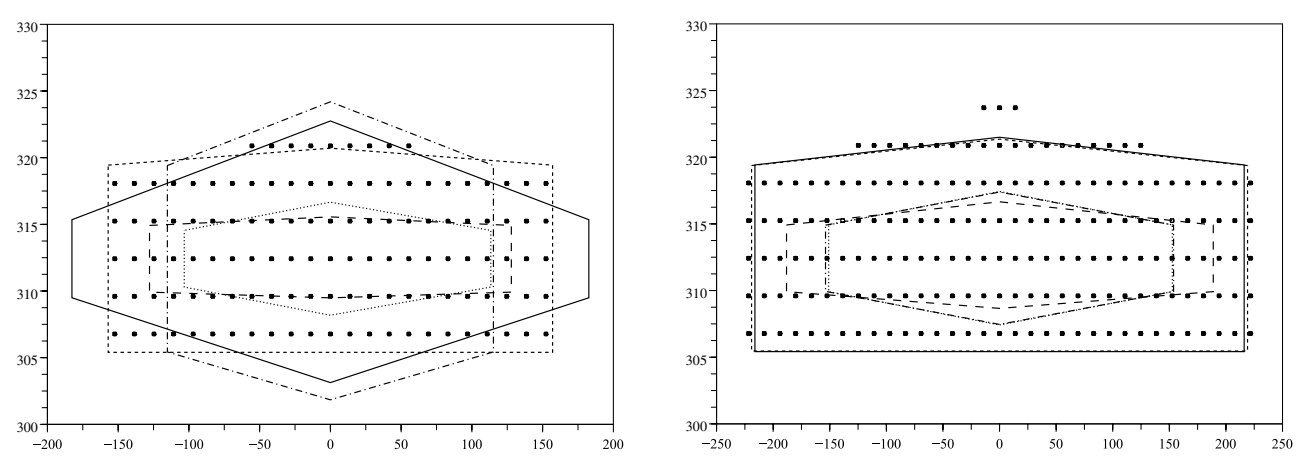

$1 / \mathrm{r}=1.9574464$

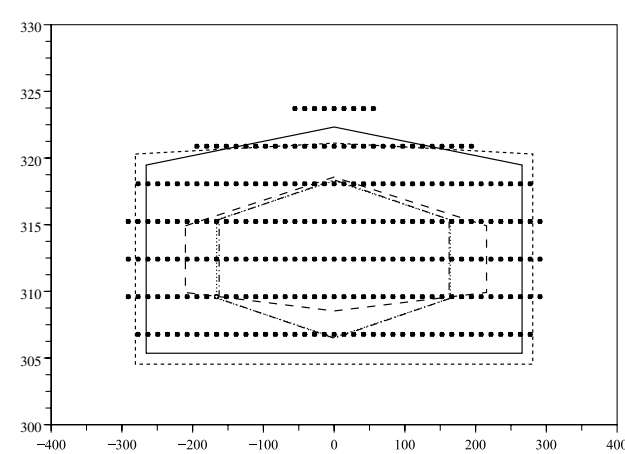

$1 / \mathrm{r}=2.4626093$

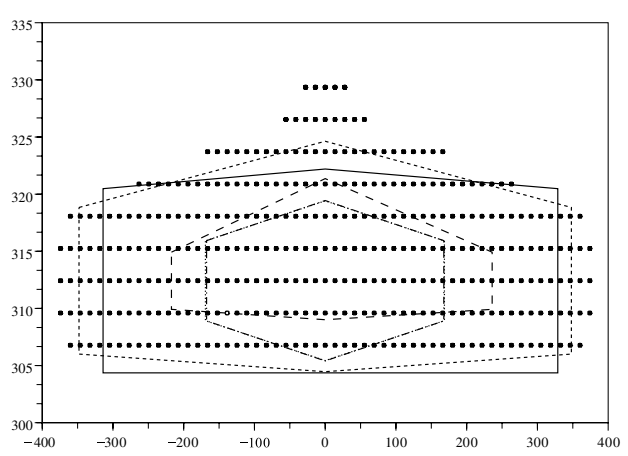

Figure 9. Asymmetric height growth: fracture foot-print and corresponding estimated polygon shape obtained from the perfect moment data, data with 5\% noise and using the harmonic moments estimated from displacement data using different orders of decomposition.

from the harmonic moments are highly correlated. In particular, both the fracture half-length and height are highly correlated to the fracture opening, especially for the case of the inverted moments. As a result, the over-estimation of the fracture opening is counter-balanced by an under-estimation of the polygonal dimensions (lower fracture half-length and height). Such 


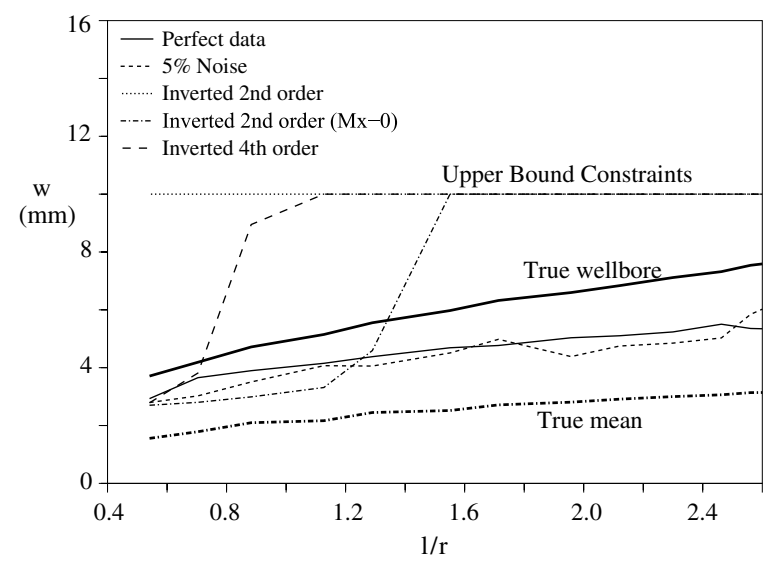

Figure 10. Asymmetric height growth: different estimates of fracture width of the polygon model as well as the true width at the well-bore (maximum) and the true mean value across the fracture.

non-uniqueness in the problem can be ameliorated: by exploiting the moment expansion to achieve a more judicious placement of the sensing array in order to obtain more accurate harmonic moments; or by including a priori information (e.g. the target layer height $H_{l}$ can be estimated from well logs or an ansatz for the functional form of the aperture distribution in the $y$ direction can be made assuming a state of plane strain prevails for vertical cross-sections).

\section{Conclusions}

The loss of resolution with distance, that is intrinsic to the elliptic elasticity PDE, renders the use of parametrized crack models difficult: either the data are sampled too far to contain any information on the crack shape besides the volume, while, if the data are sampled too close to the fracture, an incorrect model can infer unrealistic results while still fitting the data. The multipole harmonic moment decomposition presented in this paper provides more flexibility: no ad hoc assumptions on the loading and geometry are made and the order of the decomposition can be adapted depending on the measurement location. Because the multipole expansion approach is able to separate the contributions of each of the harmonic moments according to their radius of influence, it provides significantly improved estimation of the fracture volume from measured data over a far larger range of $\ell / r$ ratios than any finite fracture model. Moreover, accurate estimates of the first-order moments can be used to detect fracture height or fracture length asymmetry - an important feature in oil and gas applications where asymmetry is symptomatic of breakout of the fracture into another sedimentary layer.

Repetitive inversion with different orders of decomposition can provide an indirect way to estimate the fracture length-scale if the results for the harmonic moments differ. Different assumptions on the fracture symmetries can be also incorporated in the decomposition by assuming that some of the odd-order moments are zero. Ideally, a model selection procedure can also be applied in order to test these different assumptions and to detect the most probable decomposition for a particular configuration.

The inversion of shape from moments is an active field of research. In this paper, we have considerably simplified the problem to the identification of the dimensions of a polygonal model. Our approach can easily be enriched by including a priori information and by using more sophisticated algorithms. However, this 'second phase' of the analysis will only give 
accurate results if more precise values of the higher harmonic moments can be obtained from the measured deformation data. The more flexible harmonic moment expansion may be used to obtain more accurate moment estimates via optimal placement of the sensing array. This might, however, be difficult to achieve in practice.

The framework developed here for the mapping of cracks is closely related to the theory of eigenstrain $[15,32]$. It is directly applicable to the mapping of a-seismic faults, and can easily be extended to the detection of eigenstrain inclusions in a homogeneous medium-a problem of particular interest in the identification of residual stresses [19]. Applications to other problems involving elliptic operators is also possible.

\section{Acknowledgments}

We would like to thank Dr Rob Jeffrey for useful discussions. His constant interest in the development of new techniques for the monitoring of hydraulic fractures was a great encouragement during the course of this work. The financial support of CSIRO Petroleum is greatly acknowledged. AP also gratefully acknowledges the support of the NSERC discovery grants programme. We would like to acknowledge Schlumberger for providing the synthetic data used in the testing of the algorithms proposed herein and Dr Eduard Siebrits for a careful review of this paper.

\section{References}

[1] Adachi J, Siebrits E, Peirce A and Desroches J 2007 Computer simulation of hydraulic fractures Int. J. Rock Mech. Min. Sci. 44 739-57

[2] Andrieux A, Ben Abda A and Bui H D 1999 Reciprocity principle and crack identification Inverse Problems 15 59-65

[3] Ben Abda A, Ben Ameur H and Jaoua M 1999 Identification of 2D cracks by elastic boundary measurements Inverse Problems 15 67-77

[4] Bonnet M 2001 Boundary element based formulations for crack shape sensitivity analysis Eng. Anal. Bound. Elts 25 347-62

[5] Bonnet M and Constantinescu A 2005 Inverse problems in elasticity Inverse Problems 21 R1-50

[6] Bui H D 1994 Inverse Problems in the Mechanics of Materials: An Introduction (Boca Raton, FL: CRC)

[7] Burczynski T and Beluch W 2001 The identification of cracks using boundary elements and evolutionary algorithms Eng. Anal. Bound. Elts 25 313-22

[8] Chen D H and Nisitanu H 1993 Detection of a crack by body force method Eng. Fracture Mech. 45 671-85

[9] Clifton R J and Abou-Sayed A S 1979 On the computation of the three-dimensional geometry of hydraulic fractures Proc. SPE Symp. on Low Permeability Gas Reservoirs, Denver (SPE 7943) (Richardson TX: Society of Petroleum Engineers) pp 307-13

[10] Denison D G T, Holmes C C, Mallick B K and Smith A F M 2002 Bayesian Methods for Non-Linear Classification and Regression (New York: Wiley)

[11] Detournay E 2004 Propagation regimes of fluid-driven fractures in impermeable rocks Int. J. Geomech. 4 1-11

[12] Detournay E, Adachi J I and Garagash D I 2002 Asymptotic and intermediate asymptotic behavior near the tip of a fluid-driven fracture propagating in a permeable elastic medium Int. Conf. On Structural Integrity and Fracture (SIF) (25-28 September 2002, Perth, Australia)

[13] Du Y, Aydin A and Murdoch L 1993 Incremental growth of a shallow hydraulic fracture at a waste remediation site, oakbrook, Illinois from inversion of elevation changes Int. J. Rock Mech. Min. Sci. 30 1273-9

[14] Economides M J and Nolte M J (ed) 2000 Reservoir Stimulation 3rd edn (Chichester: Wiley)

[15] Eshelby J D 1957 The determination of the elastic field of an ellipsoidal inclusion and related problems Proc. R. Soc. A 241 376-96

[16] Evans K 1983 On the development of shallow hydraulic fractures as viewed through the surface deformation field: Part 1. Principles J. Petroleum Tech. 35 406-10

[17] Fares N and Maloof R 1998 Crack detection characterization of strain sensing grids Int. J. Solids Struct. 35 $2861-75$ 
[18] Gallego R and Rus G 2004 Identification of cracks and cavities using the topological sensitivity boundary integral equation Comput. Mech. 33

[19] Gao Z and Mura T 1989 On the inversion of residual stresses from surface displacements ASME J. Appl. Mech. 56 508-13

[20] Garagash D I and Detournay E 2000 The tip region of a fluid-driven fracture in an elastic medium ASME J. Appl. Mech. 67 183-92

[21] Garagash D I and Detournay E 2005 Plane-strain propagation of a fluid-driven fracture: small toughness solution ASME J. Appl. Mech. 72 916-28

[22] Golub G H, Milanfar P and Varah J 1999 A stable numerical method for inverting shape from moments SIAM J. Sci. Comput. 21 1222-43

[23] Hills D A, Kelly P A, Dai D N and Korsunsky A M 1996 Solution of crack problems: the distributed dislocation technique Solid Mechanics and its Applications vol 44 (Dordrecht: Kluwer)

[24] Hori A 2004 Application of spectral decomposition of Green's function to linear inverse problem Eng. Anal. Bound. Elts 28 183-93

[25] Keat W D, Larson M C and Arthur Verges M 1998 Inverse method of identification for three-dimensional subsurface cracks in a half-space Int. J. Fracture 92 253-70

[26] Larson M C, Arthur Verges M and Keat W D 1999 Nondestructive identification of three-dimensional embedded cracks in finite bodies by inversion of surface displacements Eng. Fracture Mech. 63 611-29

[27] Lecampion B and Gunning J 2007 Model selection in fracture mapping from elastostatic data Int. J. Solids Struct. 44 1391-408

[28] Lecampion B, Jeffrey R and Detournay E 2005 Resolving the geometry of hydraulic fractures from tilt measurements Pure Appl. Geophys. $1622433-52$

[29] Margonari M and Bonnet M 2005 Fast multipole method applied to the coupling of elastostatic BEM with FEM Comput. Struct. 83 700-17

[30] Mindlin R D 1953 Force at a point in the interior of a semi-infinite solid Proc. 1st Midwestern Conf. on Solid Mechanics (Urbana, IL: University of Illinois) p 111

[31] Mitchell S L, Kuske R and Peirce A P 2007 An asymptotic framework for the analysis of hydraulic fractures: the impermeable case J. Appl. Mech. 74365

[32] Mura T 1982 Micromechanics of Defects in Solids (The Hague: Martinus Nijhoff)

[33] Peirce A P 2005 Localized Jacobian ILU preconditioners for hydraulic fractures Int. J. Numer. Methods Eng. $651935-46$

[34] Peirce A P and Siebrits E 2001 The scaled flexibility matrix method for efficient solution of boundary value problems in 2d and 3d layered elastic media Comput. Methods Appl. Mech. Eng. 190 5935-56

[35] Peirce A P and Napier J A L 1995 A spectral multipole method for efficient solution of large-scale boundary element models in elastostatics Int. J. Numer. Methods Eng. 38 4009-34

[36] Rongved L 1957 Dislocation over a bounded plane area in an infinite solid ASME J. Appl. Mech. 24 252-4

[37] Siebrits E and Peirce A P 2002 An efficient multi-layer planar 3D fracture growth algorithm using a fixed mesh approach Int. J. Numer. Methods Eng. 53 691-717

[38] Storn R and Price K 1997 Differential evolution: a simple and efficient heuristic for global optimization over continuous space J. Global Optim. 11 341-59

[39] Stravoulakis G E and Antes H 1997 Nondestructive elastostatic identification of unilateral cracks through BEM and neural networks Comput. Mech. 20

[40] Warpinski N R 2000 Analytic crack solutions for tilt fields around hydraulic fractures J. Geophys. Res. 105 23463-78

[41] Weikl W, Andra H and Schnack E 2001 An alternating iterative algorithm for the reconstruction of internal cracks in a three-dimensional solid body Inverse Problems 17 1957-75

[42] Wright C A, Davis E J, Minner J F, Weijers L, Schnell E J and Hunter S P 1998 Surface tiltmeter fracture mapping reaches new depths_-10,000 feet, and beyond SPE Regional Meeting on Low Permeability Reservoirs (SPE 39919)

[43] Yoshida K, Nishimura N and Kobayashi S 2001 Application of fast multipole Galerkin boundary integral equation method to elastostatic crack problems in 3D Int. J. Numer. Methods Eng. 50 525-47 